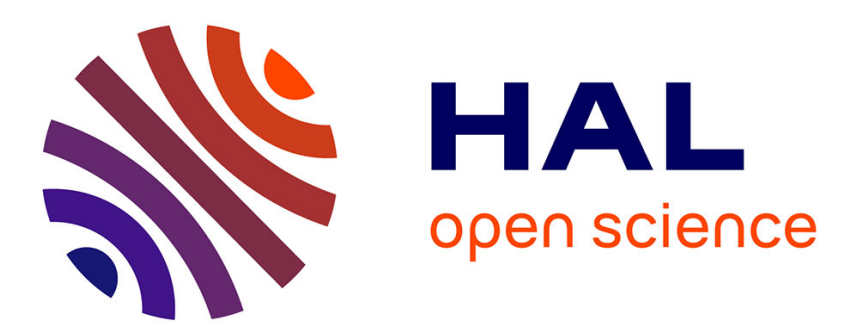

\title{
Mobilité électronique dans les verres semi-conducteurs à base de V 205
}

\author{
M. Michaud, P. Pineau, J. Livage, R. Collongues
}

\section{To cite this version:}

M. Michaud, P. Pineau, J. Livage, R. Collongues. Mobilité électronique dans les verres semiconducteurs à base de V 2O5. Revue de Physique Appliquée, 1977, 12 (5), pp.715-719. 10.1051/rphysap:01977001205071500 . jpa-00244232

\section{HAL Id: jpa-00244232 https://hal.science/jpa-00244232}

Submitted on 1 Jan 1977

HAL is a multi-disciplinary open access archive for the deposit and dissemination of scientific research documents, whether they are published or not. The documents may come from teaching and research institutions in France or abroad, or from public or private research centers.
L'archive ouverte pluridisciplinaire HAL, est destinée au dépôt et à la diffusion de documents scientifiques de niveau recherche, publiés ou non, émanant des établissements d'enseignement et de recherche français ou étrangers, des laboratoires publics ou privés. 


\title{
MOBILITÉ ÉLECTRONIQUE DANS LES VERRES SEMI-CONDUCTEURS A BASE DE $\mathrm{V}_{2} \mathrm{O}_{5}$
}

\author{
M. MICHAUD, P. PINEAU, J. LIVAGE et R. COLlONGUES \\ Spectrochimie du solide, ERA 387, E. N. S. C. P., 11, rue Pierre et Marie-Curie, 75005 Paris, France
}

\begin{abstract}
Résumé. - Les verres à base de $\mathrm{V}_{2} \mathrm{O}_{5}$ sont des semi-conducteurs à faible mobilité. Les porteurs de charge sont de petits polarons se déplaçant par sauts entre des sites $\mathrm{V}^{4+}$ et $\mathrm{V}^{5+}$. La mobilité de ces polarons est étudiée par Résonance Paramagnétique Electronique en fonction de la structure et de la composition du verre. On montre que cette mobilité est beaucoup plus faible dans l'oxyde amorphe que dans l'oxyde cristallisé (localisation d'Anderson) et qu'elle peut être modifiée par l'addition en faible quantité d'oxydes étrangers. Selon la position qu'occupe l'impureté dans le réseau vitreux, la mobilité peut être augmentée $(\mathrm{Li}, \mathrm{Na}, \mathrm{Cu})$ ou diminuée $\left(\mathrm{P}_{2} \mathrm{O}_{5}\right)$. Tous les verres étudiés sont obtenus par hypertrempe de l'anhydride vanadique fondu.
\end{abstract}

\begin{abstract}
V}_{2} \mathrm{O}_{5}$ containing glasses are known to be low mobility semiconductors. This property arises from the hopping of small polarons from $\mathrm{V}^{4+}$ to $\mathrm{V}^{5+}$ ions. The mobility of the polarons has been studied by ESR. It depends on the structure and the composition of the glass.

In this paper, we show that the hopping frequency decreases when the disorder increases, leading to a localization of the charge carriers (Anderson localization). The hopping frequency also varies when adding small quantities of foreign oxides. It increases with lithium oxide and decreases with $\mathrm{P}_{2} \mathrm{O}_{5}$. All glasses have been obtained by splat cooling.
\end{abstract}

L'anhydride vanadique $\mathrm{V}_{2} \mathrm{O}_{5}$ se dissocie partiellement à la fusion en perdant de l'oxygène. Il en résulte un oxyde non-stoechiométrique dans lequel les lacunes d'oxygène sont compensées par la formation d'ions $\mathrm{V}^{4+}$.

Les verres à base de $\mathrm{V}_{2} \mathrm{O}_{5}$ sont des semi-conducteurs à faible mobilité [1-8]. Cette propriété est due à la présence simultanée au sein du matériau, d'ions vanadium à différents degrés d'oxydation, $\mathrm{V}^{4+}$ et $\mathrm{V}^{5+}$ dont les configurations électroniques sont respectivement $3 \mathrm{~d}^{1}$ et $3 \mathrm{~d}^{0}$. L'électron « $3 \mathrm{~d} » d u \mathrm{~V}^{4+}$ induit localement une déformation du réseau et les porteurs de charge sont en fait des petits polarons qui se déplacent par saut d'un site $\mathrm{V}^{4+}$ à un site ${ }^{5+}$ [9-12].

Les propriétés semi-conductrices de ces verres ont fait l'objet de nombreux travaux mais, $\mathrm{V}_{2} \mathrm{O}_{5}$ ne donnant pas spontanément de phase vitreuse, il faut lui ajouter un oxyde formateur tel que $\mathrm{P}_{2} \mathrm{O}_{5}$. C'est pouquoi tous les systèmes étudiés jusqu'à présent contenaient toujours moins de $90 \%$ en mole de $\mathrm{V}_{2} \mathrm{O}_{5}$.

Les techniques d'hypertrempe développées depuis quelques années pour l'obtention de métaux ou alliages métalliques amorphes [13] ont été étendues aux oxydes $[14,15]$. Elles nous ont permis d'obtenir de l'anhydride vanadique pur sous forme amorphe [16].

Dans ce travail, nous avons montré que la Résonance Paramagnétique Electronique, pouvait être une bonne technique de caractérisation de ces verres semi-conducteurs. Elle permet de préciser l'ordre à courte distance autour du vanadium et apporte des informations semiquantitatives sur la mobilité des électrons. L'étude qui est présentée porte essentiellement sur l'oxyde $\mathrm{V}_{2} \mathrm{O}_{5}$ pur amorphe obtenu par hypertrempe et sur l'influence de l'addition, en faible quantité, d'oxydes étrangers.

1. Préparation des oxydes amorphes par hypertrempe. - L'oxyde, ou le mélange d'oxydes, est placé dans un creuset de platine chauffé par induction et dont le fond est percé d'un trou suffisamment petit pour permettre au liquide de tenir par capillarité (Fig. 1). Lorsque l'oxyde est fondu, on le projette

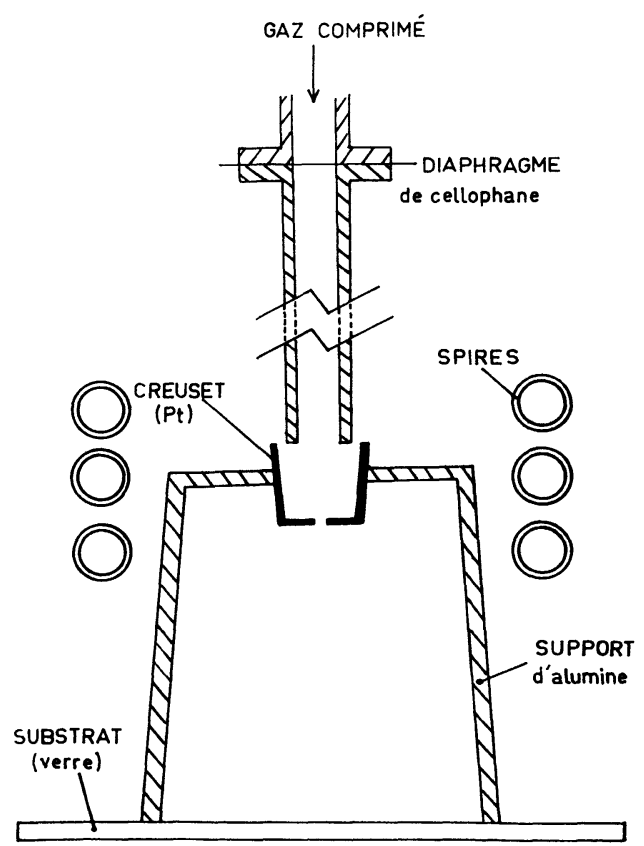

Fic. 1. - Dispositif utilisé pour réaliser l'hypertrempe de l'oxyde de vanadium $\mathrm{V}_{2} \mathrm{O}_{5}$. 
violemment sur une plaque de verre placée quelques centimètres au-dessous du creuset. Cette projection est obtenue en libérant brusquement un jet d'argon sous pression dans un tube d'alumine placé juste audessus du creuset. On réalise ainsi des vitesses de trempe pouvant atteindre $10^{5}{ }^{\circ} \mathrm{C} / \mathrm{s}$ et on recueille sur la plaque des paillettes bleu-noir, à reflets brillants, de quelques microns d'épaisseur.

L'oxyde de vanadium obtenu est amorphe aux rayons X. Il cristallise par chauffage vers $200^{\circ} \mathrm{C}$ en donnant $\mathrm{V}_{2} \mathrm{O}_{5}$ orthorhombique [16], dont la structure représentée sur la figure 2 a été décrite par Bachmann et coll. [17].
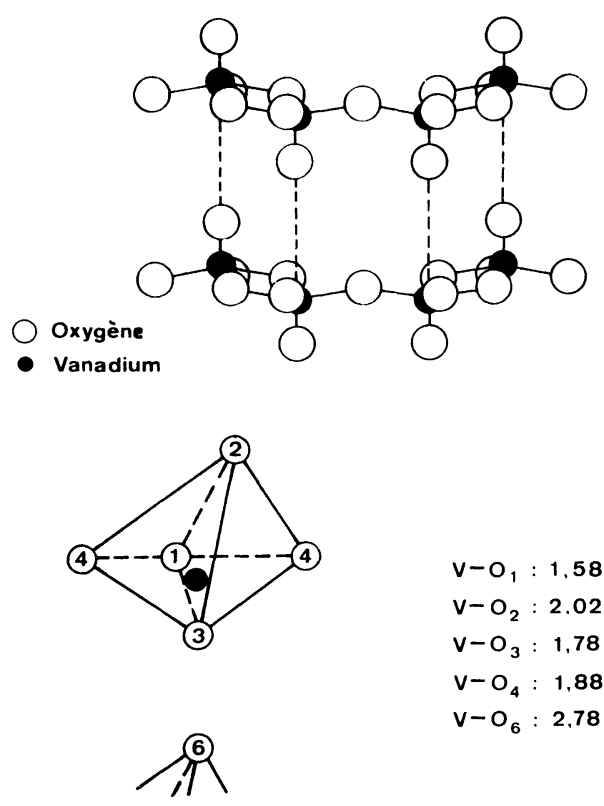

$$
\begin{aligned}
& V-O_{1}: 1,58 \AA \\
& V-O_{2}: 2.02 \AA \\
& V-O_{3}: 1,78 \AA \\
& V-O_{4}: 1,88 \AA \\
& V-O_{6}: 2,78 \AA
\end{aligned}
$$

FIG. 2. - Structure de $\mathrm{V}_{2} \mathrm{O}_{5}$ orthorhombique d'après [17].

2. Description des spectres de résonance paramagnétique électronique. - L'étude $\mathrm{du} \mathrm{V}^{4+}$ dans les verres, a été effectuée par RPE en bande $X$, sur un appareil Jeol ME 3X. Les expériences à basse température ont été réalisées en faisant circuler de l'azote gazeux, refroidi à la température désirée, à travers la cavité.

La figure 3 représente le spectre obtenu avec un échantillon d'oxyde pur et amorphe obtenu par hypertrempe. Il présente les nombreuses raies de structure hyperfine caractéristiques d'un ion $\mathrm{V}^{4+}(S=1 / 2$, $I=7 / 2$ ) placé dans un site à forte distorsion axiale. Ce spectre a été interprété en fonction de l'hamiltonien classique :

$$
\begin{aligned}
\mathscr{H}=g_{\|} \beta H_{z} S_{z}+g_{\perp} \beta\left(H_{x} S_{x}+H_{y} S_{y}\right)+ & + \\
& +A_{\|} S_{z} I_{z}+A_{\perp}\left(S_{x} I_{x}+S_{y} I_{y}\right)
\end{aligned}
$$

dans lequel $z$, axe principal des tenseurs $g$ et A, correspond à la direction de distorsion du site dans lequel se trouve le vanadium.

Le tableau I compare les paramètres mesurés à

\begin{tabular}{|c|c|c|c|}
\hline & $\begin{array}{l}\mathrm{V}_{2} \mathrm{O}_{5} \\
\text { monocristal }\end{array}$ & $\begin{array}{l}\mathrm{V}_{2} \mathrm{O}_{5} \\
\text { amorphe }\end{array}$ & $\begin{array}{l}\mathrm{V}_{2} \mathrm{O}_{5}-\mathrm{P}_{2} \mathrm{O}_{5} \\
92 \% 8 \%\end{array}$ \\
\hline & $1, \overline{923}$ & $1 \overline{936}$ & $1, \overline{931}$ \\
\hline & 1,986 & 1,987 & 1,984 \\
\hline$A_{\|}(\mathrm{G})$ & 180 & 201 & 201 \\
\hline (G) & 60 & 67 & 72 \\
\hline
\end{tabular}

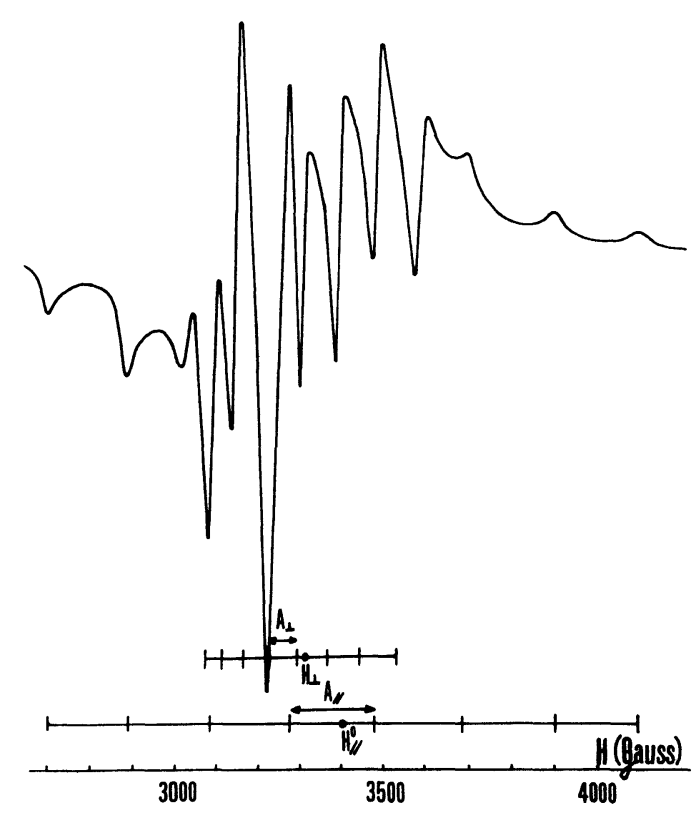

Fig. 3. - Spectre RPE de $\mathrm{V}^{4+}$ dans $\mathrm{V}_{2} \mathrm{O}_{5}$ amorphe. Température d'enregistrement $-120^{\circ} \mathrm{C}$.

\section{TABLEAU I}

Paramètres RPE de $\mathrm{V}^{4+}$ dans différents systèmes à base de $\mathrm{V}_{2} \mathrm{O}_{5}$

ceux qui avaient été obtenus avec des monocristaux de $\mathrm{V}_{2} \mathrm{O}_{5}[18,19]$.

L'analogie des facteurs " $g$ » permet de penser que l'ordre à courte distance autour du vanadium n'est guère modifié dans le verre par rapport à ce qu'il était dans la phase orthorhombique. L'accroissement notable des paramètres hyperfins montre que le couplage dipolaire entre les spins électroniques $(s=1 / 2)$ et nucléaire $(I=7 / 2)$ devient plus important dans le verre. Il est donc vraisemblable que la distance moyenne électron-noyau soit plus courte lorsque l'oxyde est amorphe [20].

La figure 4 représente les spectres de résonance paramagnétique électronique obtenus avec des verres $\mathrm{V}_{2} \mathrm{O}_{5}-\mathrm{P}_{2} \mathrm{O}_{5}$ contenant des teneurs croissantes en $\mathrm{P}_{2} \mathrm{O}_{5}$.

Ils présentent eux aussi la structure hyperfine caractéristique d'un ion $\mathrm{V}^{4+}$ en symétrie axiale et peuvent être interprétés à l'aide du même hamiltonien de spin. Les résultats des mesures sont indiqués dans le tableau I. Ils permettent de faire deux remarques : les valeurs des paramètres $" g$ " et $\| A$ " ne varient pratiquement pas, par contre on observe une diminution 


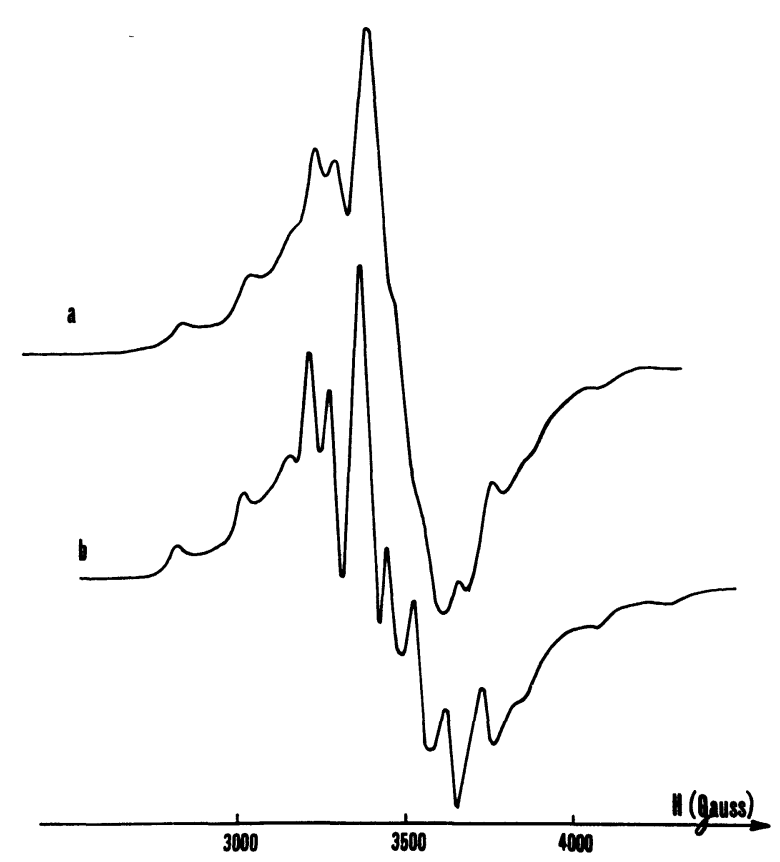

Fig. 4. - Spectres RPE de $\mathrm{V}^{4+}$ dans les verres $\mathrm{V}_{2} \mathrm{O}_{5}-\mathrm{P}_{2} \mathrm{O}_{5}$. Température d'enregistrement $-120^{\circ} \mathrm{C}: a$ ) verre contenant $2 \%$ en mole de $\mathrm{P}_{2} \mathrm{O}_{5} ; b$ ) verre contenant $8 \%$ en mole de $\mathrm{P}_{2} \mathrm{O}_{5}$.

de la largeur des raies de structure hyperfine lorsque la teneur en $\mathrm{P}_{2} \mathrm{O}_{5}$ augmente [16].

La figure 5 représente les spectres obtenus avec des verres de $\mathrm{V}_{2} \mathrm{O}_{5}$ dans lesquels on a ajouté des quantités croissantes d'oxyde de lithium. Ils montrent que contrairement au cas précédent, la largeur des raies hyperfines augmente avec la teneur en lithium. Les spectres correspondent toujours à des ions $\mathrm{V}^{4+}$ en

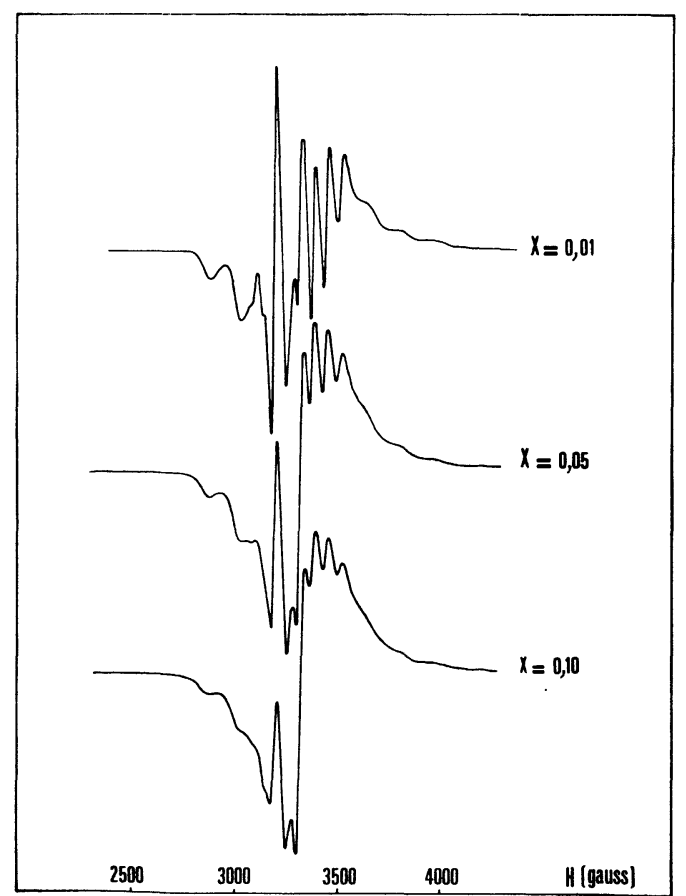

FIG. 5. - Spectres RPE de $\mathrm{V}^{4+}$ dans les verres $\mathrm{Li}_{x} \mathrm{~V}_{2} \mathrm{O}_{5}$. Température d'enregistrement $-120^{\circ} \mathrm{C}$. symétrie axiale, mais leur résolution ne permet plus de déterminer avec précision la valeur des paramètres de l'hamiltonien de spin.

2. Interprétation. - Les spectres de résonance paramagnétique électronique observés sur les échantillons précédents peuvent tous s'interpréter à l'aide du même hamiltonien de spin. Ils montrent que l'environnement de $\mathrm{V}^{4+}$ n'est pas sensiblement modifié par l'addition d'un oxyde étranger et qu'il demeure dans le verre à peu près identique à ce qu'il était dans l'oxyde orthorhombique. Ces résultats sont en accord avec ceux qui avaient été précédemment obtenus pour des verres moins riches en $\mathrm{V}_{2} \mathrm{O}_{5}[2,3,4]$.

La principale différence observée réside dans la variation de la largeur des raies de structure hyperfine. L'élargissement de ces raies peut être due à plusieurs phénomènes, en particulier :

- l'interaction dipolaire entre ions paramagnétiques voisins qui dépend de la concentration en ions $\mathrm{V}^{4+}$;

- La durée de vie des ions $\mathrm{V}^{4+}$ qui dépend du temps pendant lequel un électron $3 \mathrm{~d}$ reste localisé sur un noyau vanadium, c'est-à-dire de la mobilité électronique.

La figure 3 correspond à un oxyde amorphe dans lequel la teneur en $\mathrm{V}^{4+}$ est de $0,5 \%$ (exprimée en $\mathrm{V}^{4+} / \mathrm{V}$ total). Les raies de structure hyperfine sont relativement bien résolues ce qui indique que la mobilité électronique est faible. L'électron peut être considéré comme localisé sur un seul noyau de vanadium et sa fréquence de saut $v_{\mathrm{h}}$ est inférieure aux paramètres hyperfins exprimés en fréquence $\left(A_{\perp}=200 \mathrm{MHz}\right.$, $A_{\|}=500 \mathrm{MHz}$ ).

Selon la théorie du petit polaron, au-dessus d'une certaine température, la conductivité est essentiellement assurée par des sauts électroniques d'un site $\mathrm{V}^{4+}$ à un site $\mathrm{V}^{5+}$ voisin. Ce processus, qui fait intervenir un couplage électron-phonon, dépend de la température et la fréquence de saut peut s'exprimer par [21] :

$$
v_{\mathrm{h}}=v_{0} \exp \left(\frac{W_{\mathrm{H}}+1 / 2 W_{\mathrm{D}}}{k T}\right)
$$

$W_{\mathrm{H}}$ : est l'énergie d'activation du processus ;

$W_{\mathrm{D}}$ : est un terme qui rend compte du désordre structural. Il correspond à la différence d'énergie des niveaux « $3 \mathrm{~d}$ » des ions vanadium dans les différents sites.

Lorsque l'on passe d'une structure ordonnée (cristal) à une structure désordonnée (verre), le terme $W_{\mathrm{D}}$ augmente et la fréquence de saut $v_{\mathrm{h}}$ diminue. Elle devient très faible lorsque le désordre est grand et on observe une localisation des porteurs de charge connue sous le nom de localisation d'Anderson [22].

La figure 6 représente les spectres RPE d'un même échantillon d'oxyde $\mathrm{V}_{2} \mathrm{O}_{5}$ amorphe, mais enregistrés à différentes températures. On observe bien un élargissement progressif des raies hyperfines qui correspond à 


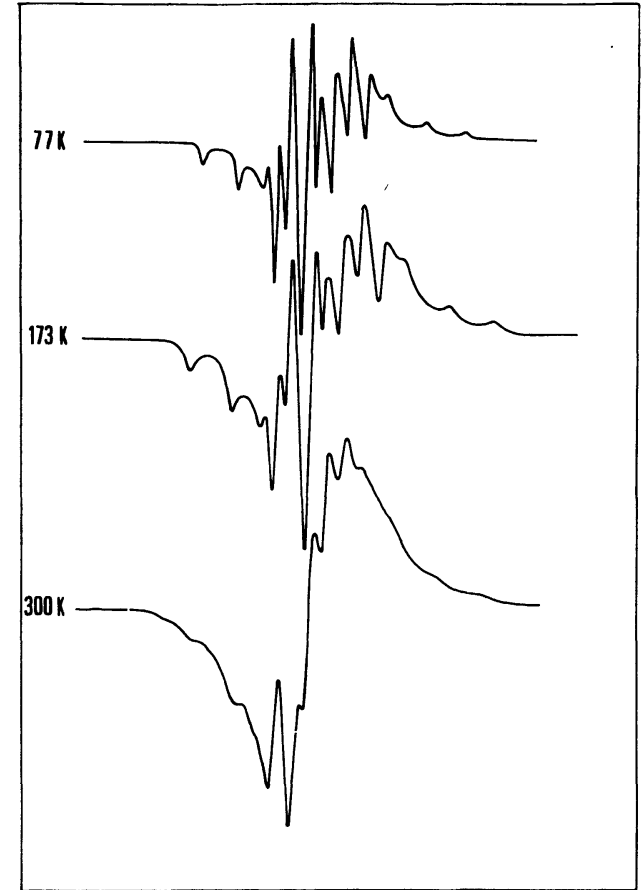

FIg. 6. - Spectres RPE de $\mathrm{V}^{4+}$ dans $\mathrm{V}_{2} \mathrm{O}_{5}$ amorphe : Températures d'enregistrement $77 \mathrm{~K}, 173 \mathrm{~K}, 300 \mathrm{~K}$.

un accroissement de la fréquence de saut lorsque la température augmente.

La figure 7 représente les spectres RPE d'un oxyde amorphe qui a subi des recuits d'une heure à différentes températures. L'analyse chimique indique que la teneur en $\mathrm{V}^{4+}$ ne varie pas, mais ce traitement permet une diffusion des ions qui s'organisent progressivement. Le désordre structural diminue et à $200^{\circ} \mathrm{C}$ on obtient la phase cristallisée orthorhombique. Tous les spectres

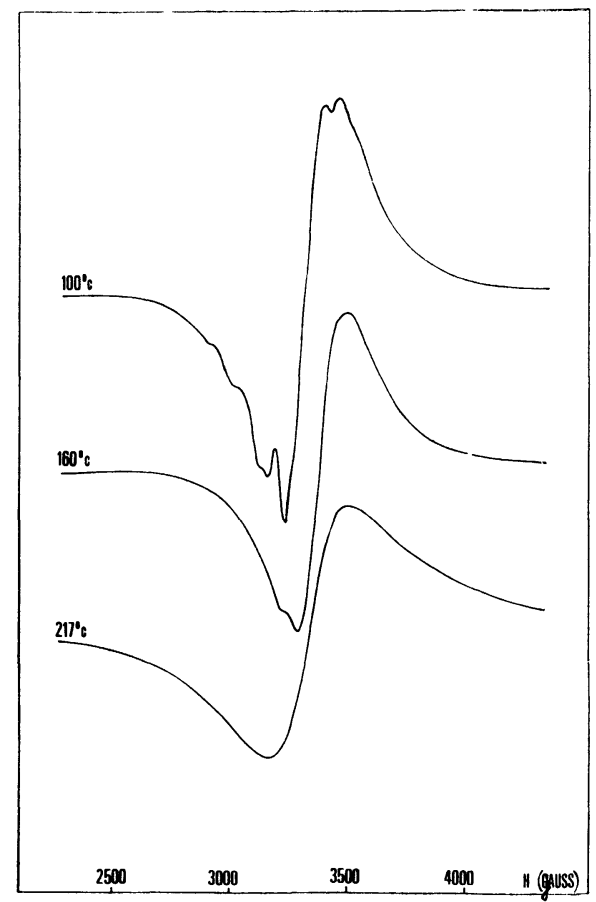

Fig. 7. - Spectres RPE de $\mathrm{V}^{4+}$ dans $\mathrm{V}_{2} \mathrm{O}_{5}$ après hypertrempe, suivie d'un recuit d'une heure à différentes températures $\left(100^{\circ} \mathrm{C}\right.$ $160^{\circ} \mathrm{C}, 217^{\circ} \mathrm{C}$ ). Température d'enregistrement $-120^{\circ} \mathrm{C}$ sont enregistrés à la même température $\left(-120^{\circ} \mathrm{C}\right)$. L'élargissement des raies est donc dû vraisemblablement à la diminution du terme de désordre $W_{\mathrm{D}}$. Pour l'oxyde cristallisé, on n'observe plus qu'une seule raie large ce qui indique que la fréquence de saut est du même ordre de grandeur que $A$., c'est-à-dire $500 \mathrm{MHz}$ environ.

La figure 4 montre que la largeur des raies hyperfines diminue lorsque l'on ajoute de l'anhydride phosphorique. Ceci ne peut être dû à un élargissement dipolaire qui augmenterait avec la teneur en $\mathrm{V}^{4+}$. L'analyse chimique montre en effet que la teneur en $\mathrm{V}^{4+}$ augmente lorsque l'on ajoute $\mathrm{P}_{2} \mathrm{O}_{5}$. Elle atteint $2 \%$ pour un verre contenant $10 \%$ en mole de $\mathrm{P}_{2} \mathrm{O}_{5}$. On observe le phénomène inverse. La variation de la largeur des raies est donc vraisemblablement due à une diminution de la mobilité électronique. Cette interprétation est en accord avec le fait que la conductivité électrique des verres $\mathrm{V}_{2} \mathrm{O}_{5}-\mathrm{P}_{2} \mathrm{O}_{5}$ diminue lorsque l'on augmente la teneur en phosphore $[2,6]$.

Dans $\mathrm{V}_{2} \mathrm{O}_{5}$, le vanadium est entouré de cinq oxygènes formant une pyramide $\mathrm{VO}_{5}$ [17]. Ces pyramides sont liées entre elles pour donner des chaînes qui forment elles-mêmes des feuillets. Un sixième oxygène placé plus loin assure la liaison entre les feuillets, (Fig. 2). Des études de résonance magnétique nucléaire ont montré que les groupements $\mathrm{PO}_{4}$ de l'anhydride phosphorique venaient prendre la place de l'oxygène situé au sommet de la pyramide $\mathrm{VO}_{5}$ (4). interrompant ainsi l'enchaînement des pyramides et s'opposant au passage de l'électron le long d'une chaîne vanadium-oxygène.

On peut imaginer ainsi, qu'au fur et à mesure que la teneur en $\mathrm{P}_{2} \mathrm{O}_{5}$ augmente, la structure en feuillet dégénère en rubans, puis en chaînes isolées. A partir d'une teneur en $\mathrm{P}_{2} \mathrm{O}_{5}$ supérieure à $10 \%$, le matériau ne peut même plus donner de phase cristallisée. Corrélativement, la conductivité et la fréquence de saut diminuent entraînant une diminution de la largeur des raies hyperfines des spectres RPE.

Par contre, lorsque l'on ajoute du lithium, on observe le phénomène inverse (Fig. 5), les raies s'élargissent progressivement. Ceci peut être dû à un accroissement de la mobilité électronique. Ces verres cristallisent en effet par chauffage en donnant des phases analogues aux bronzes de vanadium $\mathrm{Li}_{\mathrm{x}} \mathrm{V}_{2} \mathrm{O}_{5} \alpha$. On sait que dans ces bronzes, le lithium vient s'insérer entre les pyramides $\mathrm{VO}_{5}$, créant ainsi des liaisons supplémentaires, et la conductivité électrique augmente avec la teneur en lithium [23].

L'élargissement dipolaire pourrait aussi être responsable du phénomène observé, car la teneur en $\mathrm{V}^{4+}$ augmente avec l'addition de lithium. Une étude plus précise est en cours pour déterminer l'importance relative des deux phénomènes et préciser comment varie la mobilité électronique lorsque l'on ajoute du lithium. Des phénomènes d'élargissement analogues ont été observés sur des verres de $\mathrm{V}_{2} \mathrm{O}_{5}$ dans lequels on a ajouté du sodium ou du cuivre. 


\section{Bibliographie}

[1] Janakirama-Rao, V., J. Am. Ceram. Soc. 48 (1965) 311.

[2] Janakirama-Rao, V., J. Am. Ceram. Soc. 49 (1966) 605.

[3] Anderson, G. W., Compton, W. D., J. Chem. Phys. 52 (1970) 6166.

[4] Landsberger, F. R., Bray, P. J., J. Chem. Phys. 53 (1970) 2757.

[5] Caley, R. H., Murthy, M. K., J. Am. Ceram. Soc. 53 (1970) 254.

[6] Lynch, G. F., Sayer, M., Segec, S. L., Sosenblatt, G., $J$. Appl. Phys. 42 (1971) 2587.

[7] Linsley, G. S., Owen, A.E., Hayatee, F. M., J. Non Crystall. Solids 4 (1970) 28.

[8] Harper, M., McMillan, P. W., Phys. Chem. Glass 15 (1974) 148.

[9] Motт, N. F., J. Non-Crystall. Solids 1 (1968) 1.

[10] Sayer, M., Mansingh, A., Reyes, J. Y., Rosenblatt, G., J. Appl. Phys. 42 (1971) 2857.

[11] Haemers, J., Baetens, E., Vennik, J., Phys. Stat. Sol. (a) 20 (1973) 381.
[12] Sperlich, G., Urban, P., Frank, G., Z. Phys. 263 (1973) 315.

[13] Duwez, P., Willens, R. M., Klement, W., J. Appl. Phys. $31(1960) 1136$.

[14] Sarjeant, P. T., Roy, R., J. Am. Ceram. Soc. 50 (1967) 500.

[15] Kantor, P., Revcolevschi, A., Collongues, R., J. Mater. Sci. 8 (1973) 1359.

[16] Rivoalen, L., Revcolevischi, A., Livage, J., Collongues, R., J. Non-Crystall. Solids 21 (1976) 171.

[17] Bachmann, H. G., Ahmed, F. R., Barnes, W. H., $Z$. Krist. 115 (1961) 110

[18] Gillis, E., Boesman, E., Phys. Stat. Sol. 14 (1966) 337. [19] Ioffe, V. A., Patrina, I. B., Sov. Phys. Solid State 10 (1968) 639.

[20] Kahn, A., Livage, J., Collongues, R., Phys. Stat. Sol. (a) 26 (1974) 175.

[21] Austin, I. G., Mott, N. F., Adv. Phys. 18 (1969) 41.

[22] Anderson, P. W., Phys. Rev. 109 (1968) 1492.

[23] Hagenmuller, P., Progr. Solid State Chem. 5 (1971) 71. 